\title{
ELEKTIVE OPERATION
}

\section{Ist kurzfristiger Nikotinentzug schädlich?}

Eine geplante Op. ist eine gute Gelegenheit, Raucher zum Nikotinentzug zu motivieren. Wer mit dem Rauchen aufhört, senkt nachweislich sein Risiko für postoperative Komplikationen. Aber gilt das auch, wenn der Entzug erst kurz vor dem Eingriff erfolgt? Die Befürchtung ist, dass dann wegen der verstärkten Sympathikusaktivierung sogar das Gegenteil eintreten könnte. Diese Angst scheint einer Metaanalyse zu- folge jedoch unbegründet. Wer innerhalb von acht Wochen vor der Op. das Rauchen einstellt, hat zumindest nach diesen Daten kein höheres Komplikationsrisiko als Raucher. Idealerweise sollten Ärzte aber versuchen, ihre Patienten bereits einige Monate vor dem Op.-Termin zum Rauchstopp zu bewegen.

Arch Intern Med 2011; doi: 10.1001/archinternmed.2011.97

\section{KLINISCHE STUDIEN}

\section{Lebensgefährliche Rituale}

Soll ein Patient an einer klinischen Studie teilnehmen, muss - wenn der Patient selbst nicht ansprechbar ist - ein Angehöriger gefunden werden, der die Genehmigung zur Teilnahme an der Studie erteilt. Diese Prozedur mag juristisch und ethisch richtig sein, bei Patienten, die akut lebensbedrohlich erkrankt sind, kann sie lebensrettende Maßnahmen so lange verzögern, bis es zu spät ist. In der CRASH-2-Studie wurde die Wirksamkeit von Tranexamsäure bei Patienten mit schweren Kopfverletzungen ge- testet. Musste das Einverständnis von Angehörigen eingeholt werden, verzögerte dies den Beginn der Therapie um durchschnittlich eine Stunde. Das ethisch begründete Ritual der Einverständniserklärung wird unethisch, wenn es das Leben der Patienten gefährdet, schreiben Mitglieder der Clinical Trial Unit der London School of Hygiene and Tropical Medicine in einem Brief an "The Lancet".

The Lancet, 2011;

DOl: 10.1016/S0140-6736(11)60317-6

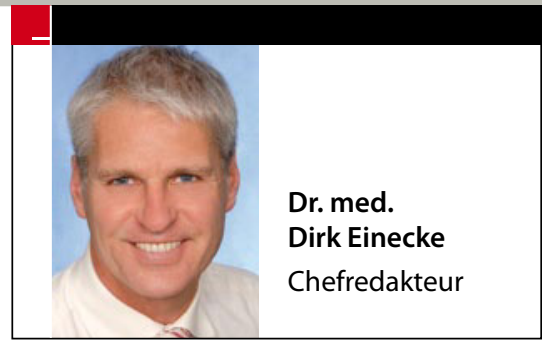

BEWEGUNGSMUFFEL IN GEFAHR

\section{Sex und Sport als Infarkttrigger}

Für bewegungsfaule Personen bringt jede ungewohnte physische Anstrengung einen kurzfristigen Anstieg des kardialen Risikos mit sich - egal, ob es sich um einen Spurt, Schneeschippen oder Sex handelt. Die Risikoerhöhung wurde jetzt in einer Metaanalyse quantifiziert: Bei episodischer körperlicher Aktivität erhöht sich das Risiko für einen Herzinfarkt um das 3,5-Fache und das für den plötzlichen Herztod um das Fünffache, jeweils im Vergleich zu den üblichen belastungsfreien Phasen. Sexuelle Aktivität bei mangelnder Fitness steigert das Infarktrisiko vorübergehend um das 2,7-Fache. JAMA 2011;305:1225-1233

\section{UNGEBREMSTER OPTIMISMUS}

\section{Schlafmangel macht risikofreudig}

\section{VERZERRTE WAHRNEHMUNG}

\section{Dicke unterschätzen ihre Leibesfülle}

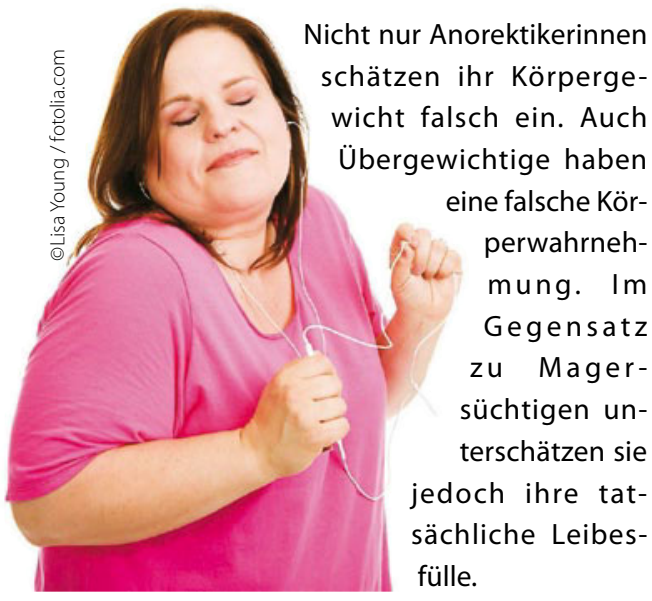

Bei einer Untersuchung von Frauen und Kindern, die sich in einem ambulanten Gesundheitszentrum in New York vorstellten, wurde nicht nur eine hohe Prävalenz von Übergewicht festgestellt: $66 \%$ der Mütter und $39 \%$ der Kinder wiesen einen BMI über 25 auf. 82\% der adipösen und 43\% der übergewichtigen Frauen (aber nur 13\% der normalgewichtigen) unterschätzten zudem ihr Gewicht. Bei den fettleibigen Kindern waren es $86 \%$. Die Hälfte der Mütter dieser Kinder wiederum hielten ihren Nachwuchs für normalgewichtig. AHA Meeting Report - Abstract P162, 23.3.2011
Unausgeschlafen sollte man keine riskanten Entscheidungen treffen. Schlafmangel aktiviert offenbar die Hirnregionen, die für optimistisches Denken verantwortlich sind, während die "Miesmacherregionen", gebremst werden. Forscher ließen 29 Probanden um Geld spielen. Nach Schlafentzug zeigten die Probanden eine erhöhte Bereitschaft, gewinnmaximierend und risikofreudig zu spielen. In der funktionellen MRT war die Aktivität der bilateralen intraparietalen Sulci und der rechten vorderen Insula (der Region, in der negative Erlebnisse verarbeitet werden) reduziert, während im ventromedialen Präfrontalcortex vergleichsweise rege gefeuert wurde. J Neurosci 2011;31(10):3712 